\title{
Mesure de la digestibilité des fourrages destinés aux chevaux par la technique des sacs de nylon
}

\author{
Nicoletta MIRAGLIA *, W. MARTIN-ROSSET ** et J.L. TISSERAND \\ Avec la collaboration de Catherine CORDELET, Elisabeth GLUNTZ et H. DUBROEUCQ ** \\ Laboratoire de Recherches de la Chaire de Zootechnie de l'ENSSAA \\ 26, bd $D^{r}$ Petitjean, F 21100 Dijon \\ * Istituto di Zootecnia, Via de Lelles, 01100 Viterbo (Italie) \\ ** INRA, Unité Elevage et Alimentation du Cheval \\ Station des Producions Bovines et Chevalines \\ Centre de Recherches de Clermont-Ferrand, Theix, F 63122 Ceyrat
}

\section{Résumé}

La digestibilité de 13 fourrages est mesurée simultanément in vivo avec des chevaux de selle adultes et par la technique des sacs de nylon sur 2 poneys mâles, adultes, munis d'une canule permanente du caecum. Le fourrage subit avant sa mise en sachet de nylon une prédigestion pepsique pendant 48 heures selon la méthode de Tilley et Terry (1963), puis le sac est introduit dans le caecum pour une durée de 24 heures. Les digestibilités de la matière sèche, matière organique et cellulose brute Weende sont calculées.

Les résultats de digestibilité en sac de nylon, comparés aux valeurs de digestibilité in vivo, montrent des corrélations satisfaisantes $(P>1 \quad p$. 100) pour la matière sèche et la matière organique, ce qui n'est pas le cas pour la cellulose brute pour laquelle il serait souhaitable d'expérimenter d'autres méthodes.

Mots clés: Chevaux, fourrages, digestibilité, sacs de nylon.

\section{Introduction}

La digestibilité des aliments destinés au cheval peut être connue directement à l'aide des tables de la valeur nutritive (N.R.C., 1978 ; FurtermitTel Tabellen, 1984 ; JarRige et Martin-Rosset, 1984). Elle peut être aussi estimée, dans le cas des fourrages, à partir de relations de prévision reliant la digestibilité à la composition chimique et notamment la teneur en parois (Axelsson, 1949 ; Olsson, 1949 ; Hintz, 1969 ; Vandernoot et Trout, 1971 ; Martin-Rosset et al., 1984). Pour améliorer la précision de la prévision, et réduire son coût en limitant le nombre d'analyses nécessaires, nous avons étudié la possibilité d'évaluer la digestibilité des fourrages à partir de leur digestion en sachets de nylon introduits dans le caecum (in sacco), ce qui évite la collecte totale des fèces. 
La méthode est déjà utilisée couramment chez le ruminant pour étudier la digestion des fourrages et prévoir leur digestibilité (van Keuren et HeinemanN, 1962 ; Hopson et al., 1963 ; Johnson, 1966 ; Demarquilly et Chenost, 1969 ; Cote et al., 1982) mais également de plus en plus chez le cheval (Applegate et Hersheberger, 1969 ; Koller et al., 1978 ; Uden et van Soest, 1982 et 1984).

La précision et la répétabilité de cette méthode dépendent de nombreux facteurs (Kercher et al., 1964 ; Tomlin et al., 1967 ; Figroid et al., 1972 ; van Hellen et Ellis, 1973 ; Uden et al., 1974 ; van Hellen et Ellis, 1977 ; Uden et van Soest, 1984) ; les plus importants sont la porosité de la toile, les dimensions des sacs, la quantité et la finesse de broyage du fourrage expérimental, le temps de séjour du sac dans le caecum et le régime alimentaire des animaux fistulés. Il existe en outre des différences liées aux animaux, au moins aussi importantes que chez les autres espèces, et à la saison (Kercher, Gallinger et Eikenberry, 1964) imputables à un éventuel effet de l'environnement et des interactions possibles entre les différents facteurs de variations.

\section{Matériel et méthodes}

\section{A. Animaux}

La digestibilité a été mesurée in vivo par collecte totale des fèces sur des chevaux de selle adultes de $500 \mathrm{~kg}$ selon un protocole décrit par ailleurs (MARTiN-Rosset et al., 1984).

La mesure de la digestibilité par la méthode des sacs de nylon a été effectuée sur 2 poneys mâles aduites et munis d'une canule permanente du caecum (Tisserand et al., 1977) car nous ne disposions pas de chevaux fistulisés.

\section{B. Fourrages}

13 fourrages ont été étudiés : 2 pailles, 7 foins de prairie naturelle de qualité très différente récoltés au $1^{\text {cr }}$ cycle $(\mathrm{n}=6)$ ou au $2^{\mathrm{c}}$ cycle $(\mathrm{n}=1)$ et 4 foins de luzerne récoltés au $1^{\text {er }}$ cycle $(n=1)$, au $2^{\mathrm{c}}$ cycle $(\mathrm{n}=2)$ ou au $3^{\mathrm{e}}$ cycle $(\mathrm{n}=1)$. Tous les fourrages ont été fanés au sol par beau temps à l'exception d'un $2^{\mathrm{e}}$ cycle de luzerne ventilé en grange.

Pendant la période de mesure de la digestibilité in vivo, des échantillons représentatifs de fourrages ont été prélevés pour la mesure ultérieure de la digestibilité de ces mêmes fourrages in sacco.

La composition chimique des fourrages étudiés est rapportée dans le tableau I. Quelques différences entre la composition des fourrages utilisés in vivo et in sacco apparaissent malgré un échantillonnage quotidien des fourrages distribués aux chevaux.

\section{Mesure de la digestibilité par la technique des sacs de nylon}

Les poneys reçoivent $5 \mathrm{~kg}$ par jour et par tête d'un foin de luzerne-dactyle de qualité moyenne (tabl. 1). L'essai ne commence qu'après 15 jours d'adaptation à ce fourrage. 
TABleau 1

Composition chimique des fourrages étudiés.

Chemical composition of tested forages.

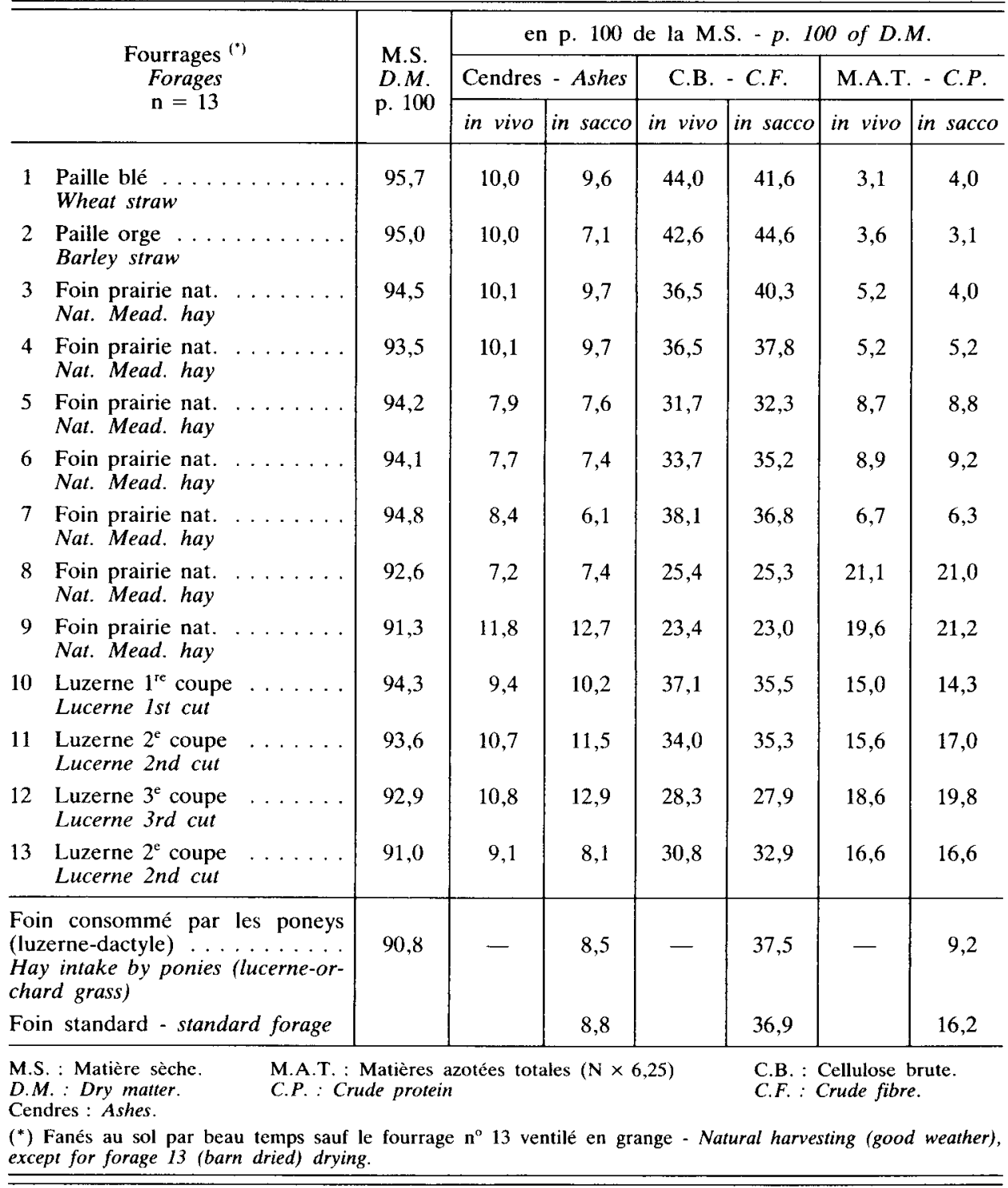

Nous n'avons pas utilisé de fourrage de référence comme témoin de chaque série de mesure in sacco car une étude antérieure réalisée au laboratoire avait montré que cela n'améliorait pas la précision de la mesure (GLUNTZ, 1984). 
$2,5 \mathrm{~g}$ de fourrage broyé avec une grille de $0,8 \mathrm{~mm}$ sont introduits dans un sac de nylon mesurant $18 \times 4 \mathrm{~cm}$ (Nylon F 100, TripetTe et Renaud, Paris). Le diamètre des mailles du tissu varie de 5 à $8 \mu$. Le sac est fermé par soudure électrique ; il est muni à son extrêmité inférieure de billes de verre afin de permettre une bonne immersion dans le caecum.

Il est prolongé à sa partie supérieure par un fil de nylon accroché à l'aide d'un œillet. Le fourrage, introduit dans le sac, subit une prédigestion avec une solution pepsique pendant 48 heures selon la $2^{\mathrm{e}}$ étape de la technique de Tilley et Terry (1963) ; après rinçage et séchage à l'air pendant 24 heures, le sac est maintenu dans le caecum pendant 24 heures, ce qui correspond au temps de séjour moyen observé dans le caecum pour les particules de fourrage (APPlegate et Hershberger, 1969) ; la mesure est répétée 5 fois pour chaque fourrage (5 jours consécutifs) par poney, soit 10 sacs par fourrage.

Les teneurs en matière sèche et en cendres sont respectivement mesurées à l'étuve à $103{ }^{\circ} \mathrm{C}$ pendant 12 heures minimum, au four à $550^{\circ} \mathrm{C}$ pendant 5 heures. La teneur en parois a été mesurée en dosant la cellulose brute par la méthode de WEENDE.

Les digestibilités in sacco de la matière sèche (d.M.S.), de la matière organique (d.M.O.) et de la cellulose brute (d.C.B.) ont été calculées selon la formule suivante :

$$
\text { Dégradabilité in sacco }=\frac{\text { M.S. disparue }(\text { pepsine }+ \text { caecum })}{\text { M.S. de départ }}
$$

Les résultats obtenus in sacco sont comparés aux résultats de la digestibilité in vivo servant de référence. Des coefficients de corrélation simple sont calculés entre les variables (FISHER et YATES, 1963).

\section{Résultats et discussion}

Le tableau 2 rapporte les valeurs des digestibilités mesurées in vivo et in sacco. La d.M.O. mesurée in sacco est plus faible que la d.M.S. $(-1,3 \pm 1,0)$ contrairement à ce que l'on observe in vivo $(+1,1 \pm 0,9)$. Les écarts observés ne sont pas significatifs et peuvent être dus, in sacco, aux caractéristiques et à l'activité de la population bactérienne des poneys (importance, composition), au temps de séjour insuffisant des sacs de nylon dans le caecum ( 24 heures) et à la digestion des matières minérales, qui doit être faible dans le caecum, encore que mal connue aujourd'hui.

La digestibilité de la cellulose brute mesurée in sacco est très inférieure à celle mesurée in vivo $(\mathrm{P}<0,05)$. La variabilité de la digestibilité de la cellulose brute mesurée in sacco est plus élevée $(\mathrm{C} . \mathrm{V} .=10$ à 53 p. 100) qu'in vivo (C.V. $<$ à 10 p. 100). L'écart est d'autant plus élevé que les fourrages sont riches en parois : pailles, mauvais foins. La flore du caecum des poneys est adaptée au fourrage unique qui leur est distribué pendant toutes les mesures alors que celle des chevaux est probablement plus spécifique à chaque fourrage étudié grâce à la période d'adaptation observée systématiquement. Tisserand et Masson (1976), Tisserand et al. (1980), Bellet (1982) ont en effet montré que la composition de la flore microbienne du caecum peut varier selon la composition du régime, la nature et la présentation des fourrages. 
TABleau 2

Résultats comparés de la digestibilité mesurée in vivo et in sacco.

Comparison of in vivo and in sacco digestibilities.

\begin{tabular}{|c|c|c|c|c|c|c|c|}
\hline & \multirow{2}{*}{$\begin{array}{c}\text { Fourrages } \\
\text { Forages } \\
\mathbf{n}=13\end{array}$} & \multicolumn{3}{|c|}{$\begin{array}{l}\text { C.U.D. in vivo } \\
\text { in vivo digestibility }\end{array}$} & \multicolumn{3}{|c|}{$\begin{array}{c}\text { C.U.D. in sacco } \\
\text { nylon bag digestibility }\end{array}$} \\
\hline & & $\begin{array}{l}\text { M.S. } \\
D . M .\end{array}$ & $\begin{array}{l}\text { M.O. } \\
O . M .\end{array}$ & $\begin{array}{l}\text { C.B. } \\
\text { C.F. }\end{array}$ & $\begin{array}{l}\text { M.S. } \\
\text { D.M. }\end{array}$ & $\begin{array}{l}\text { M.O. } \\
O . M .\end{array}$ & $\begin{array}{l}\text { C.B. } \\
\text { C.F. }\end{array}$ \\
\hline 1 & $\begin{array}{l}\text { Paille blé } \\
\text { Wheat straw }\end{array}$ & $35,0 \pm 2,7$ & $37,7 \pm 2,8$ & $38,3 \pm 3,4$ & $25,3 \pm 2,9$ & $24,6 \pm 2,6$ & $14,3 \pm 2,6$ \\
\hline 2 & $\begin{array}{l}\text { Paille orge } \ldots \ldots \\
\text { Barley straw }\end{array}$ & $34,1 \pm 2,2$ & $36,2 \pm 2,1$ & $35,6 \pm 2,3$ & $22,7 \pm 5,5$ & $22,0 \pm 5,4$ & $9,8 \pm 6,0$ \\
\hline 3 & $\begin{array}{l}\text { Foin prairie nat. } \\
\text { Nat. Mead. hay }\end{array}$ & $37,5 \pm 1,9$ & $37,9 \pm 1,9$ & $30,3 \pm 2,6$ & $41,9 \pm 1,3$ & $41,3 \pm 1,2$ & $32,9 \pm 3,0$ \\
\hline 4 & $\begin{array}{l}\text { Foin prairie nat. } \\
\text { Nat. Mead. hay }\end{array}$ & $37,4 \pm 1,7$ & $38,0 \pm 1,9$ & $30,3 \pm 0,7$ & $29,6 \pm 2,3$ & $27,4 \pm 2,5$ & $11,7 \pm 6,2$ \\
\hline 5 & $\begin{array}{l}\text { Foin prairie nat. . } \\
\text { Nat. Mead. hay }\end{array}$ & $49,3 \pm 2,4$ & $50,4 \pm 2,2$ & $39,9 \pm 3,0$ & $58,1 \pm 3,8$ & $57,9 \pm 3,4$ & $39,3 \pm 4,3$ \\
\hline 6 & $\begin{array}{l}\text { Foin prairie nat. } \\
\text { Nat. Mead. hay }\end{array}$ & $48,4 \pm 1,9$ & $49,7 \pm 1,7$ & $38,7 \pm 3,1$ & $50,7 \pm 1,3$ & $51,0 \pm 1,3$ & $37,0 \pm 2,0$ \\
\hline 7 & $\begin{array}{l}\text { Foin prairie nat. } \\
\text { Nat. Mead. hay }\end{array}$ & $44,5 \pm 2,3$ & $45,8 \pm 2,4$ & $40,1 \pm 3,0$ & $45,6 \pm 1,2$ & $45,7 \pm 1,0$ & $28,3 \pm 3,4$ \\
\hline 8 & $\begin{array}{l}\text { Foin prairie nat. } \\
\text { Nat. Mead. hay }\end{array}$ & $59,7 \pm 3,3$ & $61,2 \pm 3,2$ & $55,8 \pm 4,0$ & $53,4 \pm 3,1$ & $51,6 \pm 3,3$ & $25,3 \pm 8,6$ \\
\hline 9 & $\begin{array}{l}\text { Foin prairie nat. } \\
\text { Nat. Mead. hay }\end{array}$ & $61,7 \pm 0,4$ & $63,3 \pm 0,4$ & $60,2 \pm 1,3$ & $73,1 \pm 2,5$ & $72,9 \pm 2,6$ & $55,9 \pm 5,3$ \\
\hline 10 & $\begin{array}{l}\text { Luzerne } 1^{\mathrm{re}} \text { coupe } \\
\text { Lucerne lst cut }\end{array}$ & $54,8 \pm 2,7$ & $56,6 \pm 2,7$ & $35,3 \pm 1,1$ & $60,8 \pm 2,7$ & $59,0 \pm 2,6$ & $34,6 \pm 6,6$ \\
\hline 11 & $\begin{array}{l}\text { Luzerne } 2^{\mathrm{e}} \text { coupe } \\
\text { Lucerne 2nd cut }\end{array}$ & $56,5 \pm 1,4$ & $57,2 \pm 0,9$ & $38,6 \pm 2,7$ & $64,3 \pm 2,3$ & $61,8 \pm 2,4$ & $38,5 \pm 3,4$ \\
\hline 12 & $\begin{array}{l}\text { Luzerne } 3^{e} \text { coupe } \\
\text { Lucerne } 3 r d \text { cut }\end{array}$ & $58,8 \pm 1,3$ & $58,6 \pm 1,2$ & $40,0 \pm 1,8$ & $71,8 \pm 2,6$ & $69,5 \pm 2,9$ & $40,5 \pm 5,9$ \\
\hline 13 & $\begin{array}{l}\text { Luzerne } 2^{\mathrm{e}} \text { coupe } \\
\text { Lucerne } 2 \text { nd cut }\end{array}$ & $63,8 \pm 1,5$ & $63,0 \pm 1,6$ & $40,6 \pm 2,5$ & $58,5 \pm 3,4$ & $55,4 \pm 3,5$ & $26,4 \pm 6,5$ \\
\hline
\end{tabular}

En outre, la technique des sacs de nylon ne rend pas compte de l'ensemble des processus digestifs, notamment de l'effet de la digestion pré-caecale des fourrages sur la digestion des parois : hémicelluloses en particulier (KeYs et al., 1969), mais surtout de la dégradation encore importante des parois dans le côlon.

La cellulose brute n'est peut-être pas le meilleur critère chimique pour évaluer la digestibilité des fourrages par la méthode des sacs, même si c'est celui que nous avons pour l'instant retenu dans les relations de prévision établies à partir de mesures in vivo (MARTin-Rosset et al., 1984).

La variabilité des digestibilités mesurées in sacco (d.M.S.-d.M.O.-d.C.B.) est un peu plus élevée que celles obtenues avec la même technique par APPLEGate et Hersheberger (1969), Koller et al. (1978). Le faible nombre de fourrages étudiés $(n=3$ à 4$)$ par ces différents auteurs peut expliquer en partie la différence, de même 
que le temps de séjour des sachets dans le caecum : 24 heures ou (et) 48 heures (Koller et al., 1978).

Les différences observées peuvent être aussi liées au milieu, notamment à la saison. La dégradation in sacco de la cellulose serait plus élevée au printemps (Kercher et al., 1974 ; GlunTZ, 1984) : il pourrait exister un effet photopériodique sur l'activité microbienne du gros intestin.

L'analyse statistique rapportée au tableau 3 paraît confirmer les considérations précédentes et les résultats d'Applegate et Hersheberger, 1969. Il existe une bonne relation ( $\mathrm{P}<0,01 \mathrm{p} .100)$ entre la d.M.S. et la d.M.O. mesurée in vivo et in sacco, mais il n'y a pas de relation significative entre la d.C.B. mesurée in vivo et d.M.S., d.M.O. ou d.C.B. mesurées in sacco.

\section{TABLEAU 3}

Coefficients de corrélation simple entre les coefficients de digestibilité mesurés in vivo et in sacco $(n=13)$.

Simple correlation coefficients between in vivo and in sacco digestibility coefficients $(n=13)$.

\begin{tabular}{|c|c|c|c|}
\hline $\begin{array}{l}\text { C.U.D. in sacco } \\
\text { In sacco digestibility coefficient } \\
\text { C.U.D. in vivo } \\
\text { In vivo digestibility coefficient }\end{array}$ & $\begin{array}{l}\text { M.S. } \\
\text { D.M. }\end{array}$ & $\begin{array}{l}\text { M.O. } \\
O . M \text {. }\end{array}$ & $\begin{array}{l}\text { C.B. } \\
\text { C.F. }\end{array}$ \\
\hline $\begin{array}{l}\text { Matière sèche } \ldots \ldots \ldots \ldots \ldots \ldots \ldots \ldots \ldots \ldots \\
\text { Dry matter }\end{array}$ & $0,904^{++}$ & $0,894^{++}$ & $0,668^{+}$ \\
\hline $\begin{array}{l}\text { Matière organique } \ldots \ldots \ldots \\
\text { Organic matter }\end{array}$ & $0,897^{++}$ & $0,883^{++}$ & $0,672+$ \\
\hline $\begin{array}{l}\text { Cellulose brute } \ldots \\
\text { Crude fibre }\end{array}$ & 0,515 & 0,530 & 0,493 \\
\hline
\end{tabular}

L'objectif de ce travail était, dans un premier temps, de voir la relation entre dégradation in sacco et digestibilité in vivo et non de rechercher la méthode la plus fiable. De plus, il ne nous paraît pas opportun de proposer des équations de régression, compte tenu du petit nombre de fourrages étudiés.

\section{Conclusion}

La technique des sacs de nylon est une méthode intéressante pour évaluer et comparer, chez le cheval, la digestibilité de la matière sèche et de la matière organique des fourrages, mais pas de la cellulose brute. Cette méthode permet d'étudier l'activité du caecum et du gros intestin, notamment dans le cas des aliments cellulosiques. De plus, elle rend possible l'estimation de la qualité des fourrages (Mehrez et Orskov, 1977). 
Le principal inconvénient de cette méthode, comme d'ailleurs celui des techniques de fermentation in vitro (Tilley et Terry, 1963) est la nécessité de disposer d'animaux fistulés. C'est pourquoi cette technique nous paraît davantage destinée à l'étude de la digestion chez le cheval qu'à l'utilisation comme test de prévision de routine ; l'intervention de nombreux facteurs de variations la rend moins reproductible que des méthodes de laboratoire.

Reçu en avril 1986.

Accepté en février 1988.

\section{Summary \\ Estimation of horse forage digestibility by the nylon bag technique}

The digestibility of 13 horse forages (Table 1) was estimated by in vivo digestion trials in saddle horses and by the nylon bag technique in caecum fistulated ponies.

The forage placed in a nylon bag was pre-digested with a pepsin solution for $48 \mathrm{~h}$ according to the method of TILLEY and TERRY (1963). The bag was introduced into the caecum for a 24-h period. Dry matter (D.M.), organic matter (O.M.) and crude fibre (C.F.) digestibilities were calculated (Table 2).

Nylon bag versus in vivo digestibility values showed significant correlation coefficients (P>0.01) for D.M. and O.M. (Tableau 3), but not for C.F. Further studies are therefore required to estimate crude fibre digestibility of forages.

Key words: Horses, forages, digestibility, nylon bags.

\section{Références bibliographiques}

Axelsson J., 1949. The ability of cattle, sheep, horses and swine to digest the nutrients of the feedingstuffs. Ann. R. Agric. Coll. Swed., 16, 84-100.

Applegate C.S., Hershberger T.V., 1969. Evaluation of in vitro and in vivo cecal fermentation techniques for estimating the nutritive value of forages for equine. J. Anim. Sci., 28, 18-22.

Bellet S., 1982. Etude des effets de différents régimes sur la microflore caecale et colique du poney. Thèse Université de Dijon, $121 \mathrm{p}$.

Cote' M., Seoane J.R., Gervais P., McQueen R.E., 1982. Etudes sur la biodégradation de la matière sèche des fourrages par les méthodes in vitro et des sacs de nylon. Can. J. Plant Sci., 62, 407-413.

Demarquilly C., Chenost M., 1969. Etude de la digestion des fourrages dans le rumen par la méthode des sachets de nylon; liaison avec la valeur alimentaire. Ann. Zootech., 18, 419-436.

Figroid W., Hale W.H., Theurer B., 1972. An evaluation of the nylon bag technique for estimating rumen utilization of grains. J. Anim. Sci., 35, 113-120.

Fisher R.A., YAtes F., 1963. Statistical tables. Oliver \& Boyd Ltd. Ed., Edinburgh, 86 p.

Futtermittel Tabellen der D.L.G. für Pferde, 1984. Ed. D.L.G. Verglas, D600 Frankfurt am Main, $128 \mathrm{p}$.

Gluntz E., 1984. Essai de prévision de la digestibilité des fourrages destinés aux chevaux par la méthode des sacs de nylon. Mémoire d'études, E.N.S.S.A.A.-Dijon, 53 p. 
Hintz H.F., 1969. Review. Equine nutrition comparisons of digestion coefficients obtained with cattle, sheep, rabbits and horses. Veterinaria, 6, 45-51.

Hopson J.D., Johnson R.R., Dehority B.A., 1963. Evaluation of the dacron bag technique as a method for measuring cellulose digestibility and rate of forage digestion. J. Anim. Sci., 22 , 448-453.

JARrige R., Martin-Rosset W., 1984. Le Cheval. INRA Paris, 689 p.

Johnson R.R., 1966. Techniques and procedures for in vitro and in vivo rumen studies. J. Anim. Sci., 25, 855-875.

Kercher C.J., Gallinger D.D., Eikenberry H.D., 1964. Nylon bag technique for measuring forage value. J. Anim. Sci., 23, 880 (Abstr.).

Key S.J.E., van Soest P.J., Young E.P., 1969. Comparative study of the digestibility of forage cellulose and hemicellulose in ruminants and nonruminants. J. Anim. Sci., 29, 11-15.

Koller B.L., Hintz H.F., Robertson J.B., van Soest P.J., 1978. Comparative cell wall and dry matter digestion in the cecum of the pony and the rumen of the cow using in vitro and nylon bag techniques. J. Anim. Sci., 47, 209-215.

Martin-Rosset W., Andrieu J., Vermorel M., Dulphy J.P., 1984. Valeur nutritive des aliments pour le cheval. In: R. JARRIGE, W. MaRTIN-Rosset. Le Cheval: reproduction-sélectionalimentation-exploitation, INRA Publication-Paris, p. 689.

Mehrez A.Z., Orskov E.R., 1977. A study of the artificial fibre bag technique for determining the digestibility of feeds in the rumen. J. Agric. Sci., 88, 645-650.

National Research Council, 1978. Nutrient requirement of horse National Academy of Science. $4^{\mathrm{e}}$ Ed. Washington U.S.A., Vol. 61.33.

OLSSON N., 1949. The relation between the organic nutrients of rations and their digestibility in horses. Ann. R. Agric. Coll. Swed., 16, 644-669.

Tilley J.M.A., Terry R.A., 1963. A two stage technique for in vitro digestion of forage crops. $J$. Br. Grassl. Soc, 18, 104-111.

Tisserand J.L., Masson C., 1976. Effet de différents régimes sur l'activité biochimique dans le gros intestin du poney. $27^{\circ}$ réunion de la Féd. Europ. Zootech., 23-26 août, Zurich.

Tisserand J.L., Candau M., Houiste H., Masson C., 1977. Evolution de quelques paramètres physico-chimiques du contenu caecal d'un poney au cours du nycthémère. Ann. Zootech., 26, 429-434.

Tisserand J.L., Ottin-Pecchio M., Rollin G., 1980. Effet du mode de distribution du foin et des céréales sur l'activité cellulolytique dans le gros intestin du poney. Reprod. Nutr. Dévelop., 20, 1685-1689.

Tomlin D.C., Anderson M.J., Harris L.E., 1967. Refinements in the in vivo bag rumen technique. J. Anim. Sci., 26, 939 (Abstr.).

Uden P., Parra R., van Soest P.J., 1974. Factors influencing reliability of the nylon bag technique. J. Dairy Sci., 57, 622 (Abstr.).

UdEn P., van Soest, 1982. Comparative digestion of timothy (Phleum pratense) fibre by ruminants, equines and rabbits. Br. J. Nutr., 47, 267-272.

UDEN P., van SOEST, 1984. Investigations of the in situ bag technique and a comparison of the fermentation in heifers, sheep, ponies and rabbits. J. Anim. Sci., 58, 213-221.

Vandernoot G.W., Trout J.R., 1971. Prediction of digestive components of forages by equines. J. Anim. Sci., 33, 351-355.

van Hellen R.W., Ellis W.C., 1973. Membranes for rumen in situ digestion techniques. J. Anim. Sci., 37, 358 (Abstr.).

van Hellen R.W., Ellis W.C., 1977. Sample container porosities for rumen in situ studies. $J$. Anim. Sci., 44, 141-146.

van Keuren R.W., Heinemann W.W., 1962. Study of a nylon bag technique for in vivo estimation of forage digestibility. J. Anim. Sci., 21, 340-345. 